\title{
Phase modulation due to crystal diffraction by ptychographic imaging
}

\author{
M. Civita, ${ }^{1}$ A. Diaz, ${ }^{2}$ R. J. Bean, ${ }^{1,3}$ A. G. Shabalin,,${ }^{4,5}$ O. Yu. Gorobtsov, ${ }^{4}$ I. A. Vartanyants, ${ }^{4,6}$ and I. K. Robinson ${ }^{1,7, *}$ \\ ${ }^{1}$ London Centre for Nanotechnology and Department of Physics and Astronomy, University College, London, WC1H 0AH, United Kingdom \\ ${ }^{2}$ Paul Scherrer Institut, 5232 Villigen, Switzerland \\ ${ }^{3}$ European XFEL GmbH, 22869 Schenefeld, Germany \\ ${ }^{4}$ Deutsches Elektronen-Synchrotron, 22607 Hamburg, Germany \\ ${ }^{5}$ University of California, San Diego, California 92093, USA \\ ${ }^{6}$ National Research Nuclear University, 115409 Moscow, Russia \\ ${ }^{7}$ Condensed Matter Physics and Materials Science Division, Brookhaven National Laboratory, Upton, New York 11973, USA
}

(Received 20 September 2017; revised manuscript received 29 November 2017; published 6 March 2018)

\begin{abstract}
Solving the phase problem in x-ray crystallography has occupied a considerable scientific effort in the 20th century and led to great advances in structural science. Here we use x-ray ptychography to demonstrate an interference method which measures the phase of the beam transmitted through a crystal, relative to the incoming beam, when diffraction takes place. The observed phase change of the direct beam through a small gold crystal is found to agree with both a quasikinematical model and full dynamical theories of diffraction. Our discovery of a diffraction contrast mechanism will enhance the interpretation of data obtained from crystalline samples using the ptychography method, which provides some of the most accurate x-ray phase-contrast images.
\end{abstract}

DOI: 10.1103/PhysRevB.97.104101

\section{INTRODUCTION}

Phase problems occur widely in experimental physics whenever the amplitude of a complex wave is accessible while its phase is not. A famous example is the phase of a monochromatic X-ray wave Bragg diffracted by a crystal lattice, which is represented by a complex-valued structure factor and can reveal information about the atomic structure of the unit cell of the crystal. While the relative intensities of the various diffraction orders can be measured, their relative phases are lost by the detection process. In crystallography, the missing phases are usually derived by self-consistency with known physical properties of the crystal, for example that the electron density is real and mostly confined to the cores of the atoms in the unit cell. The development of computational "direct methods" in the 1950s has led to a revolution in crystallography because it allows direct inversion of diffraction patterns to atomicresolution real-space images of the crystal structure $[1,2]$.

Interference methods can be used to measure phases experimentally. In "three-beam" diffraction experiments, the magnitude of one structure factor becomes modulated in a characteristic way when a second Bragg peak is simultaneously excited [3]. The shape of the interference is determined by the relative phases of the two reflections involved and implicitly by that of the difference reflection [4,5], which are all coupled through the dynamical theory of x-ray diffraction [6]. This has been developed into a practical method for measuring "triplet

\footnotetext{
*Corresponding author: i.robinson@ucl.ac.uk

Published by the American Physical Society under the terms of the Creative Commons Attribution 4.0 International license. Further distribution of this work must maintain attribution to the author(s) and the published article's title, journal citation, and DOI.
}

phases" comprising the sums of the three phases involved. When enough triplets are known, the individual phases can be deduced and the structure solved [7,8]. A Bonse-Hart interferometer was used by Hirano and Momose to measure the change in phase of an x-ray beam transmitted through a diamond crystal when a Bragg reflection was excited [9]. The change in phase shift of the transmitted beam through a diffracting crystal has also been derived by a novel Kramers-Kronig method and found to have important applications to self-seeding of free-electron lasers [10]. A more direct interference method was recently proposed by Wolf [11] in which the mutual coherence function is evaluated between the direct beam and a single Bragg reflected beam. Since this contains their relative phases, the unknown phase of the Bragg reflection can be extracted. Such a method has not yet been demonstrated for $\mathrm{x}$ rays and is likely to be rather difficult to implement.

\section{PTYCHOGRAPHY EXPERIMENT}

Here we demonstrate an experimental method, somewhat related to that of Wolf [11], in which the phase of the direct beam transmitted through a crystal is shown to change whenever a Bragg peak is generated. The reflected beam needs to be strong enough to influence the forward beam, so the diffraction has to be at least at the beginning of the dynamical regime [6]. Our method measures the phase of the forward beam, using the powerful phase sensitivity of the x-ray ptychography method [12]. By imaging the phase of the crystal under investigation by ptychography [13], we can accurately measure the phase shift of the beam as it is transmitted through the crystal. By imaging a region beyond the edges of the sample, we are able to determine precisely the phase shift of the object relative to the empty region surrounding it. This phase shift, which is known to be sensitive to the x-ray refractive index and thickness, is then found to change when a Bragg peak is generated inside the crystal. 
The x-ray ptychography method is based on the use of a spatially confined, coherent beam, conventionally called the "probe," to scan an extended object at different positions. The resulting set of diffraction patterns is then collected in the far field and used to retrieve the sample's electron density. The probe position is controlled by scanning stages to always assure significant overlap between contiguous positions. In contrast to what happens in traditional coherent $\mathrm{x}$-ray diffraction imaging methods [14], ptychography incorporates this additional overlap information to reconstruct the sample image using iterative inversion algorithms [15]. It avoids the need to impose a support constraint in real space, so an image of the probe is also derived. The diversity in the measured intensities measured from partially overlapping illuminated areas, together with the knowledge of each scanning position, enables the reconstruction of the phase of an extended sample without being limited to a maximum sample size as in coherent diffractive imaging methods. Ptychography also allows a clear separation of the two contributions of sample and illuminating probe. Ptychography is a fundamentally phase-sensitive imaging technique because it measures the phase of one part of an object relative to other parts with high sensitivity. This new method has seen rapid development over the past few years and it has been used in many fields, from imaging computer chips $[16,17]$ to biological samples [18-20]. Phase sensitivity as good as 0.005 radians has been achieved [21], which is very powerful for identifying materials by their electron density level and enables material-specific segmentation of the images.

Ptychography has also been demonstrated in the case of imperfect coherence in the beam [22]. Ptychography is usually carried out in transmission to provide $2 \mathrm{D}$ projection phasecontrast images, but can be combined with tomography to give $3 \mathrm{D}$ images of the local phase which accurately represents electron density and so allows segmentation of the images into the various materials making up their composition [17,23]. Bragg ptychography, in which diffraction patterns are collected around a diffraction peak from a crystalline sample, has recently been demonstrated to be sensitive to strains [24]. This enables the exciting possibility of 3D strain imaging of extended samples [25]. Ptychography has advantages over other techniques in the accuracy of the relative phase information about the sample because this couples directly to the interference fringes seen in the coherent diffraction pattern. It has a sensitivity of $0.005 \mathrm{rad}$ [21] and its quantitative phase-shift measurements provide accurate density values in ptychographic tomography [26]. A comparison of different phase-contrast imaging methods, notably the transport of intensity equation and one based on the contrast transfer function, found $20 \%$ variations for a phantom test sample, depending on which method was used [27].

Our experiment was conducted at the coherent small-angle x-ray scattering beamline at the Swiss Light Source, Paul Scherrer Institut in Villigen, Switzerland. The experimental setup is shown in Fig. 1 and was composed of several components precisely aligned with the aid of an $\mathrm{x}$-ray camera. To focus the beam we used a $75-\mu \mathrm{m}$-diameter Fresnel zone plate (FZP) made of Au with 100-nm outermost zone width, and $1-\mu \mathrm{m}$ thickness. A central stop of 30- $\mu \mathrm{m}$ diameter was placed upstream of the FZP to block the zeroth diffraction order. Downstream of the FZP we used a $20-\mu$ m pinhole as an order-sorting aperture to select only the first diffraction order. The focal length of the zone plate was $52.7 \mathrm{~mm}$ at the working $\mathrm{x}$-ray energy of $8.7 \mathrm{keV}$.

Our sample consisted of an array of 350-nm-diameter cylindrically shaped gold nanocrystals, $100 \mathrm{~nm}$ thick, grown on a 100-nm-thick $\mathrm{Si}_{3} \mathrm{~N}_{4}$ membrane. A scanning electron microscope analysis confirmed the size of the crystals and showed a mutual spacing of approximately $1 \mu \mathrm{m}$. The Au nanocrystals were oriented with their (111) crystallographic planes normal to the substrate, but with random orientation about this. By rotating the sample membrane to an angle of $-1.79^{\circ}$, we aligned one of the off-specular $\mathrm{Au}(\overline{1} 11)$ reflections, with Bragg angle $\theta_{\mathrm{B}}=17.68^{\circ}$, but close to normal incidence. The sample was located at the focus of the FZP which provided a coherent ptychographic probe beam. The beam size obtained from profiling the reconstructed probe was $110 \mathrm{~nm}$, close to the size expected from the FZP outermost zone width of $100 \mathrm{~nm}$.

We used two Pilatus detectors [28] to collect the diffracted and transmitted intensity distributions while scanning the sample across the probe. As shown in Fig. 1, a Pilatus 2M detector was placed at $7.20 \mathrm{~m}$ from the sample in the transmission direction, while a smaller Pilatus $100 \mathrm{k}$ was placed at the reflected Bragg direction at an angle $2 \theta_{\mathrm{B}}=35.4^{\circ}$ and at a distance of $1.03 \mathrm{~m}$. Bragg ptychography [24,25] was found to be possible with these data, but not investigated further in this paper.

\section{IMAGING RESULTS}

We started our experiment by centering a crystal in the beam and optimizing its precise Bragg angle to collect the highest intensity in our reflection detector. Then we defined a series of angles centered around the Bragg angle and we performed a ptychographic acquisition at each of them. Each ptychographic data set consisted of a series of 458 acquisitions of $0.5 \mathrm{~s}$ in a grid of concentric circles with a radial step size of $0.05 \mu \mathrm{m}$ covering a field of view of $1.2 \times 1.2 \mu \mathrm{m}$ [20]. Since the sample is a weak phase object, the 458 patterns are all very similar and differ mainly in their outer regions, appearing as blue in the example shown in Fig 1. Flares appear there whenever the probe crosses the sample edges, which provides the information for the crystal shape to be imaged. The change with Bragg angle is very hard to see in the raw data. Ptychographic reconstructions were done using a difference map algorithm described in Refs. [29,30]. For the reconstructions, a region of the Pilatus 2M detector of 192 $\times 192$ pixels of $172 \mu \mathrm{m}$ was used, corresponding to a pixel size of $31.2 \mathrm{~nm}$ in the reconstructed image. Reconstructed phase images were then corrected for a phase offset and ramp which are inherent ambiguities arising from the ptychographic reconstructions [31]. Because there was a small drift on the sample position at different angles, the reconstructed images were registered with subpixel precision [32]. In Fig. 2(a) we show a reconstructed phase image of the nanocrystal, while Figs. 2(b) and 2(c) show the amplitude and phase of the reconstructed probe. The crystal has the expected shape for dewetting from the substrate. The probe has the complex Airy pattern structure expected for the focus of a Fresnel zone plate, with approximately constant phase over its central lobe. 


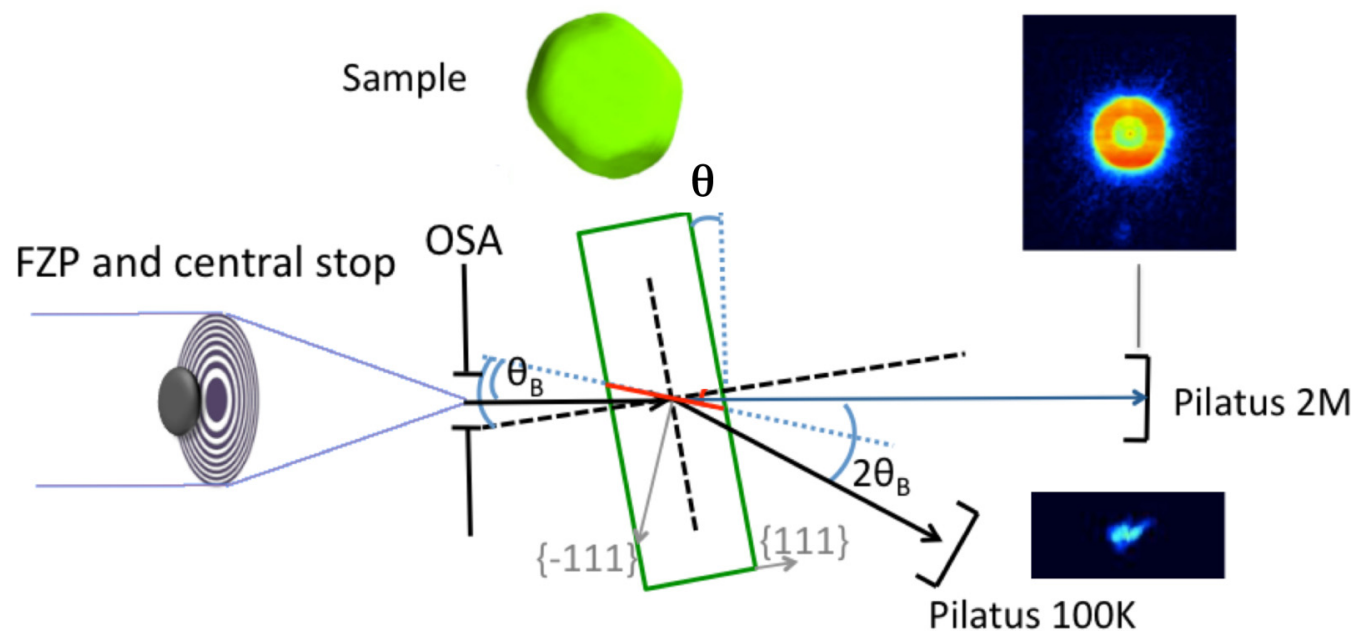

FIG. 1. Setup schematic. A Fresnel zone plate is used to focus the beam. The Au nanocrystals were placed at the focus with their (111) crystal planes oriented at the angle $\theta_{\mathrm{B}}$ with respect to the incoming beam. A 2D detector is placed in the forward direction and another $2 \mathrm{D}$ detector in the direction of the Bragg reflection.

In Fig. 3(a) we show line profiles of the phase images along the dashed-dotted line indicated in Fig. 2(a) as a function of diffraction angle around the Bragg reflection. We observe a phase shift between the Au particle and the surrounding area of about $0.18 \mathrm{rad}$, which is the value expected for a crystal thickness of $102 \mathrm{~nm}$, assuming a refractive index of $\delta=4.0 \times$ $10^{-5}$ for $\mathrm{Au}$ at $8.7 \mathrm{keV}$ [33]. More importantly, we observe that this phase shift varies slightly for different angles $\theta$ while rocking the crystal around the Bragg angle, $\theta_{\mathrm{B}}$. In Fig. 3(b) we plot the total phase shift as a function of $\Delta \theta=\theta-\theta_{\mathrm{B}}$. We determined these values by computing the difference between the integrated values inside and outside the particle, using the regions indicated in Fig. 2(a) with dotted lines. The error bars were taken as twice the standard deviation of the pixel values considered in the computation, which takes into account inhomogeneities present in the crystal and its surroundings.

\section{KINEMATICAL MODEL}

A preliminary explanation of why there is a change in phase shift can be provided by consideration of the phase of the diffraction scattered by a simple slab of crystal illuminated with a plane wave in the specular Bragg geometry. Considering the relative phases due to the path-length difference of each diffracting plane, we can sum the complex amplitude of the scattered beam as [34]

$$
R_{N}(Q)=A \sum_{j=0}^{N-1} e^{i Q d j}=A \frac{1-e^{i Q d N}}{1-e^{i Q d}},
$$

where $N$ is the number of planes in the crystal, $A \ll 1$ is a dimensionless cross-section parameter (which can be used as a scale factor in fitting to experimental data), and $d$ is the plane spacing, which is $0.235 \mathrm{~nm}$ for $\mathrm{Au}(111)$. The $j=0$ to $N-1$ individual layers are therefore at positions $z=d j$. $Q$ represents the component of momentum transfer along the (111) surface-normal direction, as calculated from the beam geometry.

As is customary for diffraction from a thin slab, the experimental intensity rocking curve should agree with the square amplitude of Eq. (1). Because of the inclined sample geometry corresponding to the $\overline{1} 11$ reflection in Fig. 1, the momentum transfer $Q$ appearing in Eq. (1) is the component perpendicular to the sample surface along the 111 direction, which we denote $Q_{\perp}$. The sample geometry in reciprocal space is shown in Fig. 4, which defines the angle $\theta$ between the incident wave
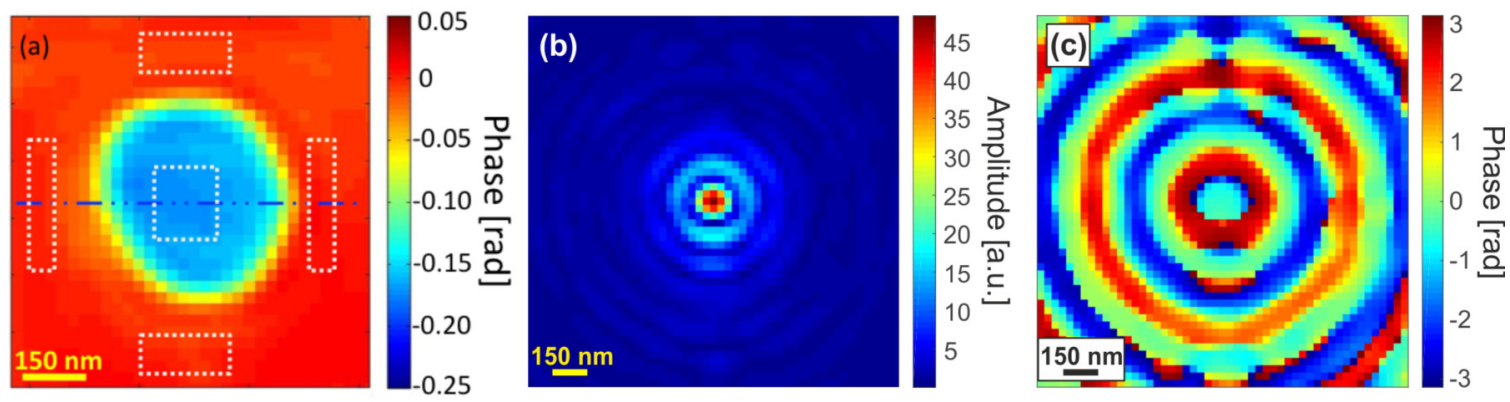

FIG. 2. (a) Reconstructed phase image of a nanocrystal at an angle close to the (111) Bragg reflection. The regions marked with dotted and dashed lines were used for quantitative analysis (see text for details). (b) Reconstructed amplitude of the illumination probe, showing a focused beam of 110-nm size. (c) Reconstructed phase of the illumination probe. 

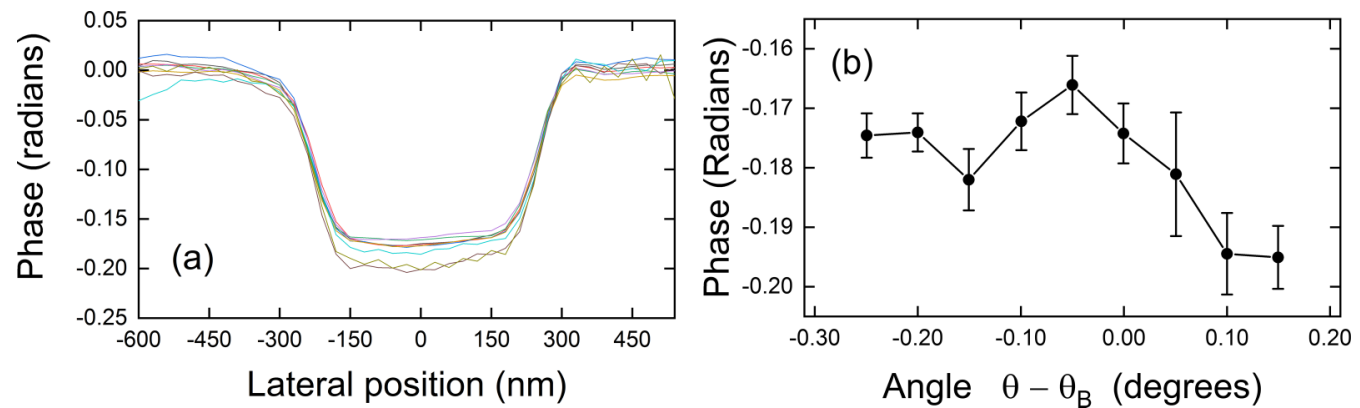

FIG. 3. (a) Phase profiles along the dashed-dotted line in Fig. 2(a) for different angles $\theta$ around the Bragg reflection. (b) Phase-shift difference between the particle and the outside region as a function of the Bragg angle, $\theta$.

vector $k_{\mathrm{i}}$ and the $Q_{\perp}$ axis, the surface normal 111 direction of the sample. The exit wave vector $k_{\mathrm{f}}$ is at an angle $\alpha$. The wave-vector magnitude, $k=2 \pi / \lambda$, where $\lambda=0.142 \mathrm{~nm}$ is the $\mathrm{X}$-ray wavelength used in the experiment. The perpendicular component $Q_{\perp}$ can be evaluated from the geometry in Fig. 4 as

$$
\begin{aligned}
Q_{\|} & =k \sin \alpha-k \sin \theta \\
Q_{\perp} & =k \cos \theta-k \cos \alpha \\
& =k\left[\cos \theta-\sqrt{1-\left(\frac{Q_{\|}}{k}+\sin \theta\right)^{2}}\right],
\end{aligned}
$$

where $Q_{\|}=2 \pi / d \sqrt{ } 8 / 3$ is fixed by the reciprocal lattice geometry. The agreement of the measurement with Eq. (1), using the perpendicular component, $Q_{\perp}$, from Eq, (2), is shown in Fig. 5(a). The data points in Fig. 5(a) were obtained by integrating the intensities collected on the reflection detector. These are overlaid with the function $\left|R_{\mathrm{N}}\left(Q_{\perp}\right)\right|^{2}$ calculated for thickness $N d=100 \mathrm{~nm}$, after adjustment of the arbitrary scale factor, $A$, and a small offset of the $\theta$ angle by $0.14^{\circ}$ (accounting for the sample alignment on the tilt stage), to compare experimental and theoretical data, as shown in Fig. 5(a) in the red and blue lines, respectively. This shows the agreement expected for a finite slab of material, following the form of a $\operatorname{sinc}^{2}$ function, in which the width of the peak is inversely related to the slab

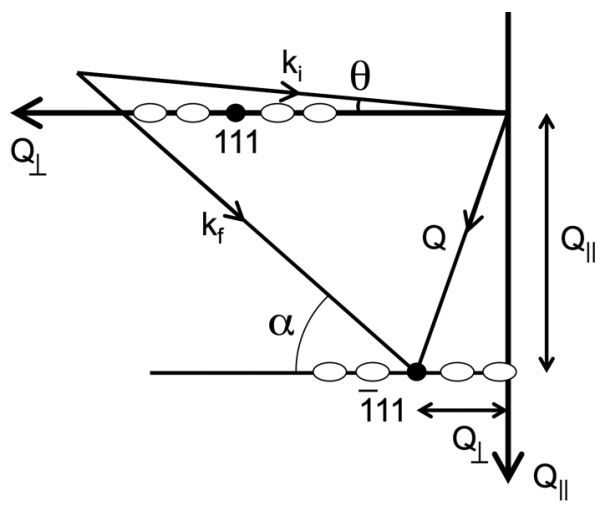

FIG. 4. Reciprocal space construction of the perpendicular component of momentum transfer needed to calculate the sample reflectivity profile. The orientation is the same as the experimental schematic layout in Fig. 1. The thickness fringes of the sample slab, perpendicular to the surface, are shown schematically as ellipses. thickness, which was independently determined to be $102 \mathrm{~nm}$ by the phase-shift measurement.

What is addressed in this study is the phase of expression (1) for the reflected beam, which is plotted in Fig. 5(b). The phase can be seen to ramp strongly within the fringes of the sinc function, including its central maximum around the Bragg angle, and to jump by $\pi$ upon crossing each node. We note that the steepness of the ramping behavior depends on the choice of origin used to derive Eq. (1), but the phase reversal crossing the nodes does not. Another example is the dynamical diffraction consideration of the Darwin curve, where the reflected phase changes from 0 to $\pi$, linearly with angle within the total reflection region, resulting in x-ray standing waves when it

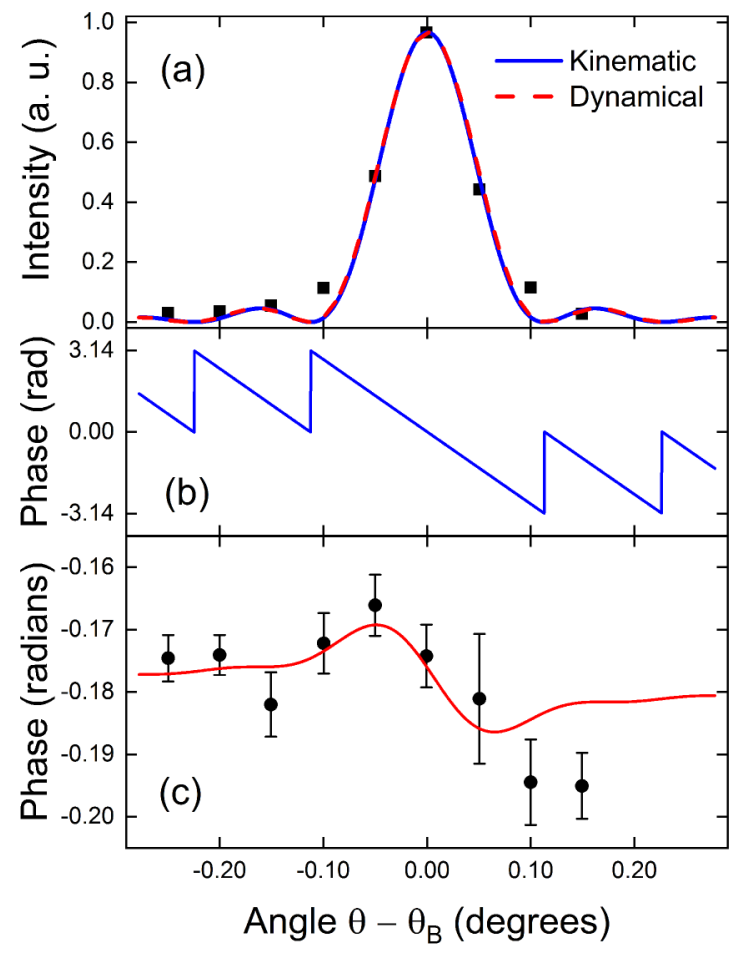

FIG. 5. (a) Rocking curve of diffracted intensity points with kinematical fitting curves from Eq. (1) shown as a blue line and the dynamical calculation as a red line. (b) Corresponding phase of the diffracted intensity in Eq. (1). (c) Measured transmitted phase shift, identical data to those shown in Fig. 3(b), together with the dynamical curve, calculated from Eq. (3) by the method of Gorobtsov and Vartanyants [36]. 
interferes with the incoming beam [35]. We also note that the phase of the reflected beam is difficult to measure directly because this would require some phase reference, usually obtained by wave interference. Instead, we turn our attention to the phase of the transmitted beam, which is the one accessible by ptychography.

\section{DYNAMICAL MODEL}

Central to the result reported here is how this strong modulation of the reflected phase in turn influences the transmitted beam, as seen by Hirano and Momose [9]. It is this phase which is measured in our ptychography experiment. In the forward direction there is a portion of the incident wave front passing beside the sample crystal, which serves as a reference wave for establishing the phase shift of the wave passing through to the sample. To understand the phase shift of the transmitted wave due to the creation of the Bragg reflection requires use of the dynamical diffraction theory to account for the redirection of energy from one beam to the other as it passes the crystal. In our case, this is more closely approximated by the Laue geometry $[6,35]$ for which the energy oscillates between the transmitted and reflected beams over the extinction length, which is $610 \mathrm{~nm}$ for $8.5-\mathrm{keV} x$ rays in gold [36]. Our sample is much thinner than this length, so only the beginning of the oscillation is probed.

Gorobtsov and Vartanyants [36] have analyzed this situation using the dynamical theory by use of the Takagi-Taupin method [37,38]. Plane-wave illumination was assumed in this work, which is an approximation for the central lobe of the reconstructed probe in our experiment shown in Fig. 2(c). In the simpler version of their result, called the quasikinematical approximation, the degree of phase modulation depends on the Bragg reflection $h$ as $\chi_{\mathrm{h}} \chi_{\overline{\mathrm{h}}}$, where $\chi_{\mathrm{h}}$ is the complex dielectric susceptibility of the crystal [39]. While a quadratic dependence is expected of the phase modulation on the crystal thickness, we are in the "thin-crystal" limit where the quasikinematical approximation applies. Given that the dynamical theory is quantitative and that all the coupling coefficients are known for $\mathrm{Au}$, the diffraction geometry is known, so the amplitude and phase of both the transmitted and reflected beams can be calculated from the crystal slab thickness. In the quasikinematical limit, the phase shift of the transmitted beam $\delta \phi_{\text {dyn }}$ can be approximated [36]:

$$
\begin{aligned}
\delta \phi_{\text {dyn }}(\Delta \theta)= & -\frac{(k d N)^{2}}{8 \cos 2 \theta_{B}}\left[\Re\left(\chi_{h} \chi_{\bar{h}}\right) \frac{1}{\Omega}\left[1-\cos \Omega\left(\frac{\sin \Omega}{\Omega}\right)\right]\right. \\
& \left.+\Im\left(\chi_{h} \chi_{\bar{h}}\right) \frac{1}{\Omega} \sin \Omega\left(\frac{\sin \Omega}{\Omega}\right)\right] \\
\Omega \simeq & \frac{k d N \sin 2 \theta_{B}}{2 \cos 2 \theta_{B}} \Delta \theta
\end{aligned}
$$

where all the symbols are previously defined.

Figure 5(a) shows the reflectivity calculated using the dynamical approach [36] for a 100-nm-thick Au crystal slab in our geometry, described below. One single parameter, a scale factor, has been adjusted to overlay with the measured data points. Good general agreement is found with the data as well as the simple reflectivity function of Eq. (1). The side fringes were not resolved in the measurement because, in the oblique sample geometry, the integration of the diffraction pattern on the detector washes out most of the fringes, but they can be clearly seen in both theory curves. The transmitted wave is a complex function whose phase, relative to that of the incident beam, is measured in our experiment. The dynamical calculation for $\delta \phi_{\text {dyn }}$ from Eq. (3) is superimposed on our experimental results in Fig. 5(c). While the agreement is generally good, it is worth noticing that there is some discrepancy with the last two points of the phase-shift curve. This mismatch is believed to be connected with the fact that the sample was seen to be drifting slightly from its original position, which negatively affected our reconstructions at the last two angular positions. This result demonstrates that the phase of the beam transmitted through a finite crystal varies across the rocking curve and this causes an angle-dependent phase shift in the transmitted beam which is understood from the dynamical theory [36].

The detailed theoretical predictions assumed the incident radiation to be a plane wave with $8.7 \mathrm{keV}$ of photon energy, calculating the diffraction from a laterally infinite $100-\mathrm{nm}$ thick crystalline plate of gold. Strictly the beam is focused, rather than plane wave, but since most of the intensity is in the center, where it can be considered planar, we conclude that it is safe to model the phase shift with an incident plane wave to a good approximation. The crystalline planes were inclined by about $18^{\circ}$ to the surface, which corresponds to the case of roughly normal incidence for the (111) reflection. The complex wave field on the exit surface of the crystal was obtained by two different approaches based on dynamical theory of x-ray diffraction [6,35]. In the first method, the Takagi-Taupin equations $[37,38]$ were solved numerically on a two-dimensional integration network [40]; in the second one an analytical solution based on the Green functions approach [41] was used. The complex diffracted and transmitted amplitudes for different angle deviations from the Bragg condition were obtained by these two methods as shown in Figs. 5(a) and 5(c). Results of both approaches were consistent with each other within the thickness of the lines in Fig. 5. The rocking curve in Fig. 5(a) has the expected form of a sinc ${ }^{2}$-function that corresponds to the kinematical limit discussed in Eq. (1) above. In Fig. 5(c) the angular dependence of the phase of the transmitted wave is presented. This curve reveals small variations from the constant refraction phase shift of $0.18 \mathrm{rad}$ which are clearly visible. These variations of about $0.016 \mathrm{rad}$ in total originate from coupling between the transmitted and diffracted beams. We emphasize that only tabulated values of the material constants were used in the calculation with the only adjustable parameters being the height of the reflectivity curve and the Bragg angle. Both the magnitude and its modulation amplitude are predicted correctly by the dynamical theory.

\section{DISCUSSION}

In summary, using a ptychographic approach [15] we have measured how the phase of an X-ray beam transmitted through a thin crystal changes when a diffracted beam is generated inside the crystal. The observed phase change is found to agree with predictions of the dynamical theory of diffraction [6,35], evaluated numerically for the specific sample thickness. For the case we considered, the phase change follows a derivative line 
shape, advanced on one side of the rocking curve and retarded on the other.

This work also demonstrates the high sensitivity with which the phase can be measured using the x-ray ptychography approach. This quality arises directly from the high coherence of the x-ray beam used. The beam traversing the crystal is coherently mixed with the beam that passes around it, resulting in a deviation of the phase of the transmitted beam. The pattern of these deviations is reconstructed as a phase contrast image self-consistently for all angular positions of the sample.

\section{ACKNOWLEDGMENTS}

We thank Sergei Gorelick for the fabrication of the Fresnel zone plate, Manuel Guizar-Sicairos for discussions and assistance during the measurements, and Xavier Donath for technical support at the beamline. The work was supported by the EPSRC Grant No. EP/I022562/1, "Phase modulation technology for X-ray imaging.” Work at Brookhaven National Laboratory was supported by the U.S. Department of Energy, Office of Science, Office of Basic Energy Sciences, under Contract No. DE-SC00112704.
[1] G. Bricogne, Acta Crystallogr. A 40, 410 (1984).

[2] H. Hauptman and J. Karle, Acta Crystallogr. 6, 136 (1953).

[3] H. Juretschke, Phys. Rev. Lett. 48, 1487 (1982).

[4] S. Chang, H. King, M. Huang, and Y. Gao, Phys. Rev. Lett. 67, 3113 (1991).

[5] Q. Shen, Phys. Rev. Lett. 80, 3268 (1998).

[6] A. Authier, Dynamical Theory of X-ray Diffraction (Oxford University Press, Oxford, 2003).

[7] Q. Shen and R. Colella, Acta Crystallogr. A 44, 17 (1988).

[8] E. Weckert and K. Hummer, Acta Crystallogr. A 53, 108 (1997).

[9] K. Hirano and A. Momose, Phys. Rev. Lett. 76, 3735 (1996).

[10] G. Geloni, V. Kocharyan, and E. Saldin, J. Mod. Opt. 58, 1931 (2011).

[11] E. Wolf, Phys. Rev. Lett. 103, 075501 (2009).

[12] J. M. Rodenburg, A. C. Hurst, A. G. Cullis, B. R. Dobson, F. Pfeiffer, O. Bunk, C. David, K. Jefimovs, and I. Johnson, Phys. Rev. Lett. 98, 034801 (2007).

[13] H. Faulkner and J. Rodenburg, Appl. Phys. Lett. 85, 4795 (2004).

[14] J. Miao, P. Charalambous, J. Kirz, and D. Sayre, Nature (London) 400, 342 (1999).

[15] J. Rodenburg, Adv. Imaging Electron Phys. 150, 87 (2008).

[16] A. Schopp, P. Boye, A. Goldschmidt, S. Honig, and R. J. Hoppe, J. Microsc. 24, 9 (2011).

[17] M. Holler, M. Guizar-Sicairos, E. H. R. Tsai, R. Dinapoli, E. Müller, O. Bunk, J. Raabe, and G. Aeppli, Nature (London) 543, 402 (2017).

[18] K. Giewekemeyer, P. Thibault, S. Kalbfleisch, A. Beerlink, C. M. Kewish, M. Dierolf, F. Pfeiffer, and T. Salditt, Proc. Natl. Acad. Sci. USA 107, 529 (2010).

[19] M. Yusuf, F. Zhang, B. Chen, A. Bhartiya, K. Cunnea, U. Wagner, F. Cacho-Nerin, J. Schwenke, and I. K. Robinson, IUCrJ 4, 147 (2017).

[20] M. Dierolf, P. Thibault, A. Menzel, C. Kewish, K. Jefimovs, I. Schlichting, K. von Konig, O. Bunk, and F. Pfeiffer, New J. Phys. 12, 035017 (2010).

[21] E. Lima, A. Diaz, M. Guizar-Sicairos, S. Gorelick, P. Pernot, T. Schleier, and A. Menzel, J. Microscop. 249, 1 (2013).
[22] P. Thibault and A. Menzel, Nature (London) 494, 68 (2013).

[23] M. Dierolf, A. Menzel, P. Thibault, P. Schneider, C. Kewish, R. Wepf, O. Bunk, and F. Pfeiffer, Nature (London) 467, 436 (2010).

[24] P. Godard, G. Carbone, M. Allain, F. Mastropietro, G. Chen, L. Capello, A. Diaz, T. H. Metzger, J. Stangl, and V. Chamard, Nat. Commun. 2, 568 (2011).

[25] S. O. Hruszkewycz, M. Allain, M. V. Holt, C. E. Murray, J. R. Holt, P. H. Fuoss, and V. Chamard, Nat. Mater. 16, 244 (2017).

[26] A. Diaz, P. Trtik, M. Guizar-Sicairos, A. Menzel, P. Thibault, and O. Bunk, Phys. Rev. B 85, 020104 (2012).

[27] M. Langer, P. Cloetens, J.-P. Guigay, and F. Peyrin, Med. Phys. 35, 4556 (2008).

[28] P. Kraft, IEEE Trans. Nucl. Sci. 56, 758 (2009).

[29] P. Thibault, M. Dierolf, A. Menzel, O. Bunk, C. David, and F. Pfeiffer, Science 321, 379 (2008).

[30] P. Thibault, M. Dierolf, O. Bunk, A. Menzel, and F. Pfeiffer, Ultramicroscopy 109, 338 (2009).

[31] M. Guizar-Sicairos, A. Diaz, M. Holler, M. Lucas, A. Menzel, R. Wepf, and O. Bunk, Opt. Express 19, 21345 (2011).

[32] M. Guizar-Sicairos, S. Thurman, and J. Fienup, Opt. Lett. 33, 156 (2008).

[33] B. Henke, E. Gullikson, and J. Davis, At. Data Nucl. Data Tables 54, 181 (1993).

[34] J. Als-Nielsen and D. McMorrow, Elements of Modern X-ray Physics (Wiley, New York, 2001).

[35] B. Batterman and H. Cole, Rev. Mod. Phys. 36, 681 (1964).

[36] O. Yu. Gorobtsov and I. A. Vartanyants, Phys. Rev. B 93, 184107 (2016).

[37] S. Takagi, J. Phys. Soc. Jpn. 26, 1239 (1969).

[38] D. Taupin, Acta Crystallogr. 23, 25 (1967).

[39] O. M. Lugovskaya and S. A. Stepanov, Sov. Phys. Cryst. 36, 478 (1991).

[40] A. G. Shabalin, O. M. Yefanov, V. L. Nosik, V. A. Bushuev, and I. A. Vartanyants, Phys. Rev. B 96, 064111 (2017).

[41] V. Kohn, I. Snigireva, and A. Snigirev, Phys. Status Solidi B 222, 407 (2000). 